\title{
The Provable Virtue of Laziness in Motion Planning*
}

\author{
Nika Haghtalab ${ }^{1}$, Simon Mackenzie $^{2}$, \\ Ariel D. Procaccia ${ }^{3}$, Oren Salzman ${ }^{3}$ and Siddhartha Srinivasa ${ }^{4}$ \\ ${ }^{1}$ Microsoft Research \\ ${ }^{2}$ University of New South Wales \\ ${ }^{3}$ Carnegie Mellon University \\ ${ }^{4}$ University of Washington
}

\begin{abstract}
The Lazy Shortest Path (LazySP) class consists of motion-planning algorithms that only evaluate edges along candidate shortest paths between the source and target. These algorithms were designed to minimize the number of edge evaluations in settings where edge evaluation dominates the running time of the algorithm such as manipulation in cluttered environments and planning for robots in surgical settings; but how close to optimal are LazySP algorithms in terms of this objective? Our main result is an analytical upper bound, in a probabilistic model, on the number of edge evaluations required by LazySP algorithms; a matching lower bound shows that these algorithms are asymptotically optimal in the worst case.
\end{abstract}

\section{Introduction}

The simplest motion planning model [Halperin et al., 2017; LaValle, 2006] involves a robot system $R$ moving in a workspace $W \in\left\{\mathbb{R}^{2}, \mathbb{R}^{3}\right\}$ cluttered with obstacles $O$. Given an initial placement $s$ and a target placement $t$ of $R$, we wish to determine whether there exists a collision-free motion of $R$ connecting $s$ and $t$, and, if so, to plan such a motion.

Typically, $R$ is abstracted as a point, or a configuration, in a high-dimensional space called the configuration space $X$, where each configuration maps $R$ to a specific placement in $W$ [Lozano-Perez, 1983]. The configuration space is subdivided into the free and forbidden spaces, corresponding to placements of $R$ that are free or that intersect with an obstacle, respectively. Since the general motion-planning problem is PSPACE-hard [Hopcroft et al., 1984], a common approach is to use sampling-based algorithms [Kavraki et al., 1996; Hsu et al., 1999; LaValle and Kuffner, 1999; Karaman and Frazzoli, 2011]. These algorithms approximate $X$ via a discrete graph $G$ called a roadmap. Vertices in $G$ correspond to sampled configurations in $X$, and edges in $G$ correspond to local paths (typically straight lines). Approximately solving the motion-planning problem thus reduces to the problem of

\footnotetext{
${ }^{*}$ See the full version of this paper [Haghtalab et al., 2018] for proofs and additional material.
}

finding a collision-free shortest path in $G$ between the vertices corresponding to $s$ and $t$.

Testing if a vertex or an edge of $G$ is collision free requires one or more geometric tests called collision detection. Arguably, collision detection in general, and edge evaluation in particular, are the most time-consuming operations in sampling-based algorithms [LaValle, 2006; Choset et al., 2005]. Thus, path planning on $G$ differs from traditional search algorithms such as Dijkstra [1959] or A* [Hart et al., 1968], where the graph is typically implicit and large, but edge evaluation is trivial compared to search. Indeed, much recent work in motion planning focuses on evaluating the edges of $G$ lazily, that is, assuming that the edges do not intersect with the obstacles $O$ [Bohlin and Kavraki, 2000; Hauser, 2015; Dellin and Srinivasa, 2016; Salzman and Halperin, 2015; Choudhury et al., 2017; Mandalika et al., 2018; Mandalika et al., 2019].

In a recent paper, Dellin and Srinivasa [2016] present a unifying formalism for shortest-path problems where edge evaluation dominates the running time of the algorithm. Specifically, they define and investigate a class of algorithms termed Lazy Shortest Path (LazySP), which run any shortest-path algorithm on $G$ followed by evaluating the edges along that shortest path. The algorithms are differentiated by an edge selector function, which chooses the edges the algorithm evaluates along the shortest path. Dellin and Srinivasa show that several prominent motion-planning algorithms are captured by LazySP, using a suitable choice of this selector. Furthermore, they evaluate the algorithm empirically on a wide range of edge selectors and scenarios and show that, using this approach, nontrivial problems can be solved within seconds.

LazySP was proposed as an algorithm that attempts to minimize the overall number of edges evaluated (or queried) in the process of solving a given motion-planning problem. A natural question to ask is

... what is the query complexity of LazySP, and is

its query complexity the best possible?

In other words, can we bound the number of edges evaluated by LazySP as a function of the complexity of the roadmap $G$ ? And are there algorithms not in this class that have lower query complexity?

To address these questions, we need to explicitly model how queries are answered. We start in Section 3 by considering the deterministic setting, where the set of collision-free 
edges is determined upfront. Our first result establishes that, in this model, it is optimal to always test edges along the shortest path, i.e., in every instance there is an edge selector for which LazySP is optimal. Although the edge selector in question requires full access to the set of collision-free edges, so the real-world implications of this result are limited, it does provide a theoretical underpinning for the idea of restricting queries to shortest paths, which lies at the heart of LazySP.

In practice, we are interested in a slightly more complex model, which we call the probabilistic setting. Here, each edge is endowed with a probability of being in collision a common assumption in motion planning (see, e.g., Choudhury et al. 2016) — and we are interested in policies that minimize the query complexity, that is, policies that minimize the expected number of steps until the algorithm finds the shortest path or declares that no path exists. We first show that there are instances where LazySP is suboptimal, regardless of the edge selector. In a nutshell, we describe a delicate construction where initially querying edges that are not on the shortest path provides valuable information for subsequent queries.

So, in the probabilistic setting, LazySP is just a proxy for the (presumably intractable) optimal policy, but is it a good proxy? We answer this question in the positive. Our main result is that the query complexity of LazySP (with an edge selector satisfying a certain connectivity property) is bounded by $O(n / p)$ edge evaluations with high probability, where $n$ is the number of vertices in $G$, and $p$ is the minimum probability on any edge. We complement this result with an $\Omega(n / p)$ lower bound that holds for every algorithm that is guaranteed to be correct. We conclude that, from a worst-case viewpoint, LazySP is, in fact, (asymptotically) optimal.

\section{Model}

An instance of our problem is given by a multigraph ${ }^{1} G=$ $(V, E)$ whose set of vertices includes a source vertex $s$ and a target vertex $t$. We say that a graph $G^{\prime}=\left(V, E^{\prime}\right)$ is a subgraph of $G$ if $E^{\prime} \subseteq E$. Given a graph $G=(V, E)$ and a subgraph $G^{\prime}=\left(V, E^{\prime}\right)$ of $G$, an oracle $\mathcal{O}_{G^{\prime}}^{G}$ is a function that takes as input an edge $e \in E$ and returns YES if $e \in E^{\prime}$, and No otherwise. When $G$ is clear from the context, we suppress it in this notation.

In the path-finding problem, an algorithm ALG is given a graph $G$ and an oracle $\mathcal{O}_{G^{\prime}}$. The goal of the algorithm is to find the shortest $s$ - $t$ path in $G^{\prime}$. Since $G^{\prime}$ is not revealed to the algorithm directly, the algorithm has to query $\mathcal{O}_{G^{\prime}}$ on specific edges of $G$ to find a path. That is, $\operatorname{Alg}\left(G, \mathcal{O}_{G^{\prime}}\right)$ issues a sequence of edge queries to $\mathcal{O}_{G^{\prime}}$, and upon termination, returns an $s$ - $t$ path or decides that none exists. To ground this model in the context of a motion-planning algorithm, the graph $G$ is lazily constructed and can have edges that are in collision while the subgraph $G^{\prime}$ contains only collision-free edges.

For an algorithm to be correct, we require that it correctly identifies a shortest $s$-t path in $G^{\prime}$, or that it certify that none exists (by invalidating every possible path), for any $G$ and $G^{\prime} \subseteq G$. Therefore, a correct algorithm can only terminate

\footnotetext{
${ }^{1}$ We deal with multigraphs, rather than simple graphs, mostly for ease of exposition; see [Haghtalab et al., 2018] for a discussion of this point. We simply refer to $G$ as a graph hereinafter.
}

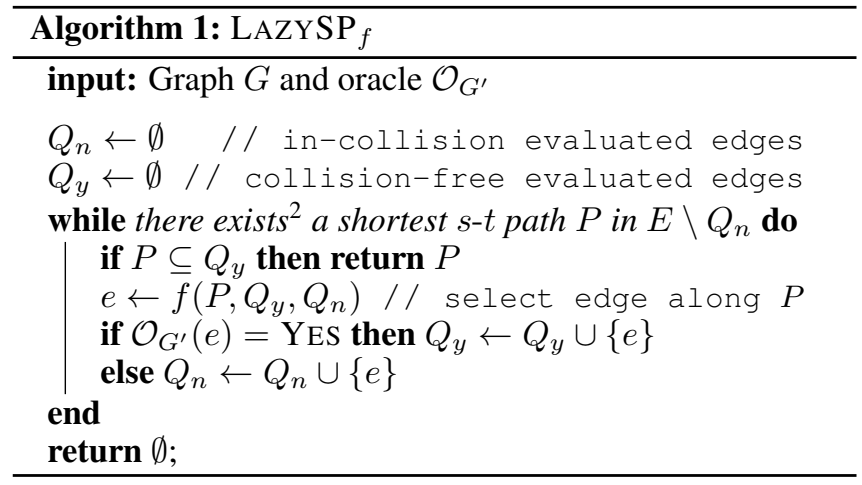

when the solution it provides continues to be correct even if the responses to unqueried edges are selected adversarially. More formally, let $Q \subseteq E$ be the set of edges queried by a correct algorithm ALG on $G$ and $\mathcal{O}_{G^{\prime}}$. Let $Q_{y}=Q \cap E^{\prime}$ and $Q_{n}=Q \backslash E^{\prime}$ be the set of queried edges that, respectively, belong and do not belong to $G^{\prime}$. Then ALG can terminate only if there is a shortest $s$ - $t$ path in $G^{\prime}$, denoted $P^{*}$, such that $P^{*} \subseteq Q_{y}$, and there is no $s$ - $t$ path in $\left(V, E \backslash Q_{n}\right)$ that is shorter than $P^{*}$. If no path exists, then ALG can terminate only if there is no $s$ - $t$ path in $\left(V, E \backslash Q_{n}\right)$.

Clearly, an algorithm that first queries all edges in $E$, thereby fully constructing $G^{\prime}$, and only then finds the shortest $s-t$ path, is a correct algorithm. However, such an algorithm may use a large number of queries, some of which may be unnecessary. In this paper, we are interested in algorithms that find a shortest $s$ - $t$ path using a minimal number of queries. We denote the number of queries that ALG makes on input $G$ and $\mathcal{O}_{G^{\prime}}$ by $\operatorname{cost}\left(\operatorname{Alg}\left(G, \mathcal{O}_{G^{\prime}}\right)\right)$.

We are especially interested in the LazySP class of algorithms, introduced by Dellin and Srinivasa [2016]. Any algorithm in the class LazySP is determined by an edge selector, which, informally, decides which edge to query on a given $s$ - $t$ path. Formally, let $\mathcal{P}$ be the set of all $s$ - $t$ paths in $G$. An edge selector is a function $f: \mathcal{P} \times 2^{E} \times 2^{E} \rightarrow E$ that takes any $s$ - $t$ path $P \in \mathcal{P}$, a subset of queried edges $Q_{y}$ that are in $E^{\prime}$, and a subset of queried edges $Q_{n}$ that are not in $E^{\prime}$, and returns an edge $e \in P \backslash Q$. For example, a Forward edge selector returns the first unqueried edge in $P$. See [Dellin and Srinivasa, 2016] for examples of edge selectors.

Given an edge selector $f$, the corresponding $\operatorname{LAZYSP}_{f} \in$ LazySP is described in Algorithm 1. At a high level, $\mathrm{LAZYSP}_{f}$, in a given time step, considers a candidate shortest $s$ - $t$ path $P$ over all those edges whose existence has not yet been ruled out by the oracle. Then, it uses the edge selector to query an unqueried edge $e \in P$. It updates the set of queried edges and repeats. At any point, if the edges of path $P$ that is currently under consideration are all verified, the algorithm terminates and returns $P$. If no viable $s$ - $t$ paths remain, the algorithm terminates and certifies that no $s$ - $t$ path exists in $G^{\prime}$.

Let us conclude this section with an example of the execution of LAZYSP with the forward edge selector, which also

\footnotetext{
${ }^{2}$ If there are multiple $s-t$ paths of the same length, the algorithm breaks ties according to a consistent tie-breaking rule.
} 


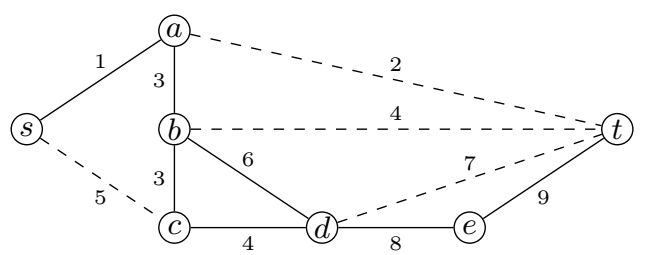

Figure 1: Example of the execution of LAZYSP with the forward edge selector. Solid edges are in $E^{\prime}$, dashed edges are in $E \backslash E^{\prime}$.

illustrates some of the terminology introduced earlier. Figure 1 shows the set of vertices $V=\{s, t, a, b, c, d, e\}$ shared by $G$ and $G^{\prime}$, as well as two types of edges: those in $E^{\prime}$, shown as solid edges, and those in $E \backslash E^{\prime}$, shown as dashed edges. The order in which edges are queried is shown as labels on the edges. This order on edge queries is induced by evaluating shortest paths in the following order: sat, sabt, scdt, sabdt, and sabdet.

\section{The Deterministic Setting}

In this section, we consider the problem of using a minimum number of edge queries to find a shortest $s$ - $t$ path, or verifying that no $s$ - $t$ path exists, when a subgraph $G^{\prime} \subseteq G$ is deterministically chosen (but not revealed to the algorithm).

In more detail, let $G=(V, E)$ be a graph, and let $G^{\prime}=$ $\left(V, E^{\prime}\right)$ be a subgraph of $G$. Recall that $\operatorname{cost}\left(\operatorname{ALG}\left(G, \mathcal{O}_{G^{\prime}}\right)\right)$ denotes the number of edge queries ALG makes on graph $G$ when oracle responses are according to graph $G^{\prime}$. Our first result asserts that the class $\mathcal{L} a z y \mathcal{S P}$ is optimal in this setting, in the sense that for any correct algorithm there is a LAZYSP algorithm (with a specific edge selector) that finds the shortest path using at most as many queries.

Theorem 1. For any graph $G$ and $G^{\prime} \subseteq G$, and any correct algorithm ALG, there exists $\mathrm{ALG}^{\prime} \in \mathcal{L}$ azySP such that

$$
\operatorname{cost}\left(\operatorname{ALG}^{\prime}\left(G, \mathcal{O}_{G^{\prime}}\right)\right) \leq \operatorname{cost}\left(\operatorname{Alg}\left(G, \mathcal{O}_{G^{\prime}}\right)\right)
$$

We can alternatively interpret Theorem 1 in a model where LAZYSP may be equipped with an omniscient edge selector that has full access to $G^{\prime}$. In particular, this omniscient edge selector can compute $Q^{*}$, which, by the way, requires solving an NP-hard variant of SET COVER. Even though the algorithm already knows $G^{\prime}$, it still has to issue queries as it must certify that $P^{*}$ is indeed the shortest path (if an $s$ - $t$ path exists).

Clearly, an omniscient edge selector is impractical. The significance of Theorem 1, therefore, is mostly conceptual. It suggests that the restriction that algorithms must always query edges on the current shortest path is not a barrier to optimality. This gives theoretical justification for the LazySP class. However, as we shall see shortly, the message is more nuanced when the outcomes of queries are randomized.

\section{The Probabilistic Setting}

Let $p \in(0,1)$ be the probability that any given edge in $G$ exists in $G^{\prime}$. We denote by $G^{\prime} \sim_{p} G$ the process of generating a random graph $G^{\prime}=\left(V, E^{\prime}\right)$ from $G$ by allowing each $e \in E$ to belong to $E^{\prime}$ with probability $p$, independently. We suppress $p$ in this notation when it is clear from the context.

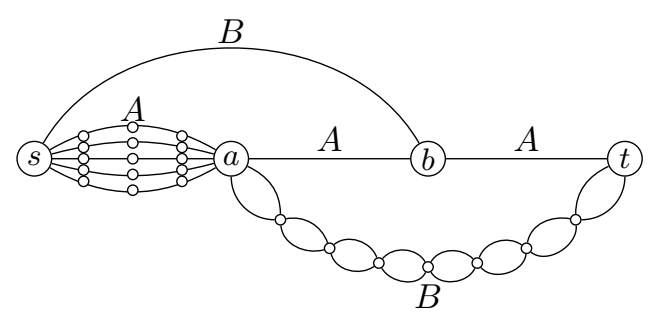

Figure 2: A graph for which no algorithm in $\mathcal{L} a z y \mathcal{S P}$ is an optimal query policy. All arcs labeled by $A$ and $B$ include multi-edge structures shown in Figures 3 and 4, respectively. For clarity, we include two examples of these structures on $s a$ and $a t$ in this figure.

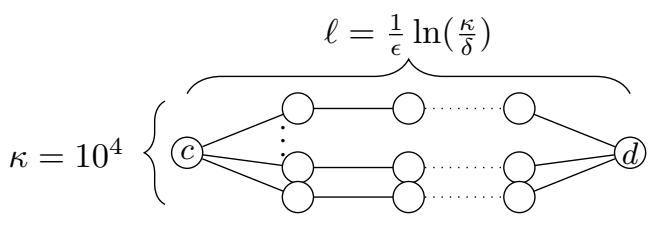

Figure 3: Structure $A$ used on arcs $s a, a b$, and $b t$ in Figure 2. We refer to one path connecting $c$ and $d$ as a "string".

Here, a subgraph $G^{\prime}=\left(V, E^{\prime}\right) \sim_{p} G$ is realized according to edge probability $p$, but it is not revealed to the algorithm. As before, the algorithm receives $G$ and $\mathcal{O}_{G^{\prime}}$ as input, and uses $\mathcal{O}_{G^{\prime}}$ to verify whether an edge exists. The goal of the algorithm is to minimize the expected number of edge queries over $G^{\prime} \sim_{p} G$, such that it correctly either (i) returns a path that is the shortest $s$ - $t$ path in $G^{\prime}$, or (ii) certifies that there is no $s$ - $t$ path in $G^{\prime}$. Note that, although the expected number of queries an algorithm issues is taken over $G^{\prime} \sim G$, the correctness condition must hold for every $G^{\prime}$.

\subsection{Suboptimality of LazySP}

Our next result asserts that the class of algorithms LazySP does not always include an optimal query policy, which minimizes the expected number of queries. At a high level, the reason behind this is that, in some graphs, querying a few edges that are not on the shortest path can identify the most important regions of the graph, which should be explored next. To see this, consider the graph in Figure 2. In this graph, the arcs marked by $A$ and $B$ each include multi-edge structures shown in Figures 3 and 4, respectively. Structures $A$ and $B$ are designed so that arcs labeled by $B$ are much longer than $A$, so any LAZYSP algorithm starts by querying the arcs labeled by $A$.

We compare the cost of any LAZYSP $\in \mathcal{L}$ azySP (for an arbitrary edge selector) to that of an algorithm ALG defined as follows. ALG first queries all the edges in the multi-edge structures $B$ on arcs $s b$ and $a t$. There are two cases:

1. A path exists in both of the structures $s b$ and $a t$, or in neither one: In this case, ALG calls LAZYSP on the original graph.

2. There is a path in exactly one of the $s b$ or at structures: Without loss of generality (by symmetry) assume that $a t$ has a path. Then, Alg queries the edges in structure $A$ on $s a, a b$ and $b t$ in order, until it verifies that at least one of these structures does not have a path or all do. 


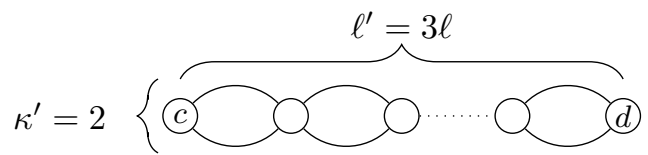

Figure 4: Structure $B$ used on arcs $s b$ and $a t$ in Figure 2.

Then, it returns the shortest $s$ - $t$ path on the edges whose existence has been verified by the queries, or certifies that no $s$ - $t$ path exists.

It is not hard to see that ALG demonstrates the required guarantees for a correct algorithm, i.e., upon its termination it correctly certifies that there is no $s$ - $t$ path or returns the shortest $s$ - $t$ path in the realized graph.

Let us provide an overview of why ALG queries fewer edges than any LAZYSP algorithm in expectation. The structures $A$ and $B$ are designed so that structure $A$ requires more queries than structure $B$. Additionally, structure $A$ almost certainly fails to have a path, while structure $B$ has a path with a probability close to $\frac{1}{2}$. Note that such a graph almost certainly does not have a path, so a large fraction of $\mathbb{E}\left[\operatorname{cost}\left(\operatorname{Alg}\left(G, \mathcal{O}_{G^{\prime}}\right)\right)\right]$ comes from the effort required to invalidate possible $s$ - $t$ paths.

In the first case of ALG (a path exists in both $a b$ and $a t$, or in neither one), it queries more edges than LAZYSP. However, we argue that ALG uses much fewer queries in its second case. The probability of existence of a path in structure $B$ is chosen so that the second case happens with significant probability (almost $\frac{1}{2}$ ), in which case the overall savings in the analysis of the second case bring down the total expected cost of ALG compared to LAZYSP.

In slightly more detail, the crux of the proof is the case where $s b$ does not have a path and $a t$ has a path (an example of the second case of ALG). To invalidate all possible $s$ - $t$ paths, it suffices to certify that structure $A$ on $s a$ does not have a path. Therefore, ALG terminates after querying only one $A$ structure, with high probability, in addition to querying two $B$ structures on $s b$ and $a t$. On the other hand, LAZYSP does not know which one of $s b$ or at has a path, so with probability at least $\frac{1}{2}$ it first queries some $A$ structure other than $s a$, in which case it has to also query and verify that no path exists in $s a$. Therefore, LAZYSP has to query $1.5 \mathrm{~A}$ structures in expectation. We design structures $A$ and $B$ so that half the cost of checking an additional $A$ structure is much larger than the initial cost that ALG invests in querying edges in two $B$ structures.

The next theorem formalizes the foregoing discussion.

Theorem 2. There is a graph $G=(V, E)$ and $p \in(0,1)$ for which the optimal query policy is not in LazySP.

\subsection{Query Complexity Bounds}

The previous section implies that algorithms in LazySP may be suboptimal in the probabilistic setting. Nevertheless, it may still be possible to give satisfying worst-case guarantees with respect to the performance of algorithms in this class. This is exactly what we do next.

Specifically, we show that any algorithm in LazySP (with an edge selector satisfying a certain property) uses $O(n / p)$

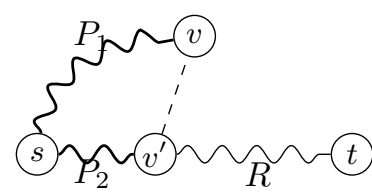

Figure 5: LAZYSP with the forward edge selector does not query an edge between two vertices in the same connected component.

queries, where $n=|V|$, w.h.p. We then show that there is a graph where no correct path-finding algorithm terminates within $\omega(n / p)$ queries. Taken together, these results show that no other algorithm can hope to do significantly better than algorithms in LazySP over all underlying graphs.

In our upper bound, we focus on edge selectors that choose an unqueried edge between two connected components formed by the validated queried edges.

Definition 1. An edge selector $f: \mathcal{P} \times 2^{E} \times 2^{E}$ is connective if for any $P \in \mathcal{P}$ and edge sets $Q_{y}$ and $Q_{n}, f\left(P, Q_{y}, Q_{n}\right)$ returns an edge $e \in P \backslash\left(Q_{y} \cup Q_{n}\right)$ that connects two connected components of the subgraph $\left(V, Q_{y}\right)$.

Let us provide an overview of why the forward edge selector (Section 2) used with a LAZYSP algorithm that breaks ties in favor of paths with more verified edges is connective. Note that at any time the set of verified edges forms a connected component around vertex $s$. Moreover, by the same reasoning behind Dijkstra [1959], if a vertex $v$ is in that connected component, the shortest $s-v$ path in $G^{\prime}$ has been found. Now, refer to Figure 5, and consider the path $P_{1}, v, v^{\prime}, R$, for two vertices $v$ and $v^{\prime}$ that are already reachable from $s$ (i.e., $P_{1} \subseteq Q_{y}$ and $P_{2} \subseteq Q_{y}$ ), and $R \subseteq E \backslash Q$. Then LAZYSP would prefer the path $P_{2}, R$, because $\left|P_{2}\right| \leq\left|P_{1}\right|+1$ (as it is the shortest path to $v^{\prime}$ ), and $P_{2}$ is fully verified. We conclude that LAZYSP with the forward edge selector never queries an edge within a connected component.

We now turn to deriving a rigorous upper bound on the number of edges queried by any LAZYSP algorithm with a connective edge selector. In terms of implications, we view this theorem as our main result.

Theorem 3. For any $\delta>0, p \in(0,1)$, graph $G$ with $n$ vertices, and a connective edge selector $f$, with probability at least $1-\delta$,

$$
\operatorname{cost}\left(\operatorname{LAZYSP}_{f}\left(G, \mathcal{O}_{G^{\prime}}\right)\right) \in O\left(\frac{n+\ln (1 / \delta)}{p}\right) .
$$

In the next theorem, we provide a matching lower bound for the number of queries that any correct path finding algorithm requires.

Theorem 4. For all $p \in(0,1)$ and $n>15$, there exists a graph $G$ with $n$ vertices such that for any correct path-finding algorithm ALG,

$$
\operatorname{Pr}_{G^{\prime}}\left[\operatorname{cost}\left(\operatorname{ALG}\left(G, \mathcal{O}_{G^{\prime}}\right)\right) \leq \frac{n-1}{2 p}\right] \leq 0.1 .
$$

\section{References}

[Bohlin and Kavraki, 2000] Robert Bohlin and Lydia E. Kavraki. Path planning using lazy PRM. In IEEE Int. 
Conf. on Robotics and Automation (ICRA), pages 521528, 2000.

[Choset et al., 2005] Howie Choset, Kevin M. Lynch, Seth Hutchinson, George Kantor, Wolfram Burgard, Lydia E. Kavraki, and Sebastian Thrun. Principles of Robot Motion: Theory, Algorithms, and Implementation. MIT Press, June 2005.

[Choudhury et al., 2016] Shushman Choudhury, Christopher M. Dellin, and Siddhartha S. Srinivasa. Paretooptimal search over configuration space beliefs for anytime motion planning. In IEEE/RSJ Int. Conf. on Intelligent Robots and Systems (IROS), pages 3742-3749, 2016.

[Choudhury et al., 2017] Shushman Choudhury, Oren Salzman, Sanjiban Choudhury, and Siddhartha S. Srinivasa. Densification strategies for anytime motion planning over large dense roadmaps. In IEEE Int. Conf. on Robotics and Automation (ICRA), pages 3770-3777, 2017.

[Dellin and Srinivasa, 2016] Christopher M. Dellin and Siddhartha S. Srinivasa. A unifying formalism for shortest path problems with expensive edge evaluations via lazy best-first search over paths with edge selectors. In Int. Conf. on Automated Planning and Scheduling (ICAPS), pages 459-467, 2016.

[Dijkstra, 1959] Edsger W. Dijkstra. A note on two problems in connexion with graphs. Numerische Mathematik, 1(1):269-271, 1959.

[Haghtalab et al., 2018] Nika Haghtalab, Simon Mackenzie, Ariel D. Procaccia, Oren Salzman, and Siddhartha S. Srinivasa. The provable virtue of laziness in motion planning. In Int. Conf. on Automated Planning and Scheduling (ICAPS), pages 106-113, 2018.

[Halperin et al., 2017] Dan Halperin, Oren Salzman, and Micha Sharir. Algorithmic motion planning. In Handbook of Discrete and Computational Geometry, Third Edition, pages 1311-1342. CRC Press, 2017.

[Hart et al., 1968] Peter E. Hart, Nils J. Nilsson, and Bertram Raphael. A formal basis for the heuristic determination of minimum cost paths. IEEE Transactions on Systems, Science, and Cybernetics, SSC-4(2):100-107, 1968.

[Hauser, 2015] Kris Hauser. Lazy collision checking in asymptotically-optimal motion planning. In IEEE Int. Conf. on Robotics and Automation (ICRA), pages 29512957, 2015.

[Hopcroft et al., 1984] John E Hopcroft, Jacob Theodore Schwartz, and Micha Sharir. On the complexity of motion planning for multiple independent objects; PSPACEhardness of the "warehouseman's problem". I. J. Robotics Res., 3(4):76-88, 1984.

[Hsu et al., 1999] David Hsu, Jean-Claude Latombe, and Rajeev Motwani. Path planning in expansive configuration spaces. Int. J. Comput. Geometry Appl., 9(4-5):495-512, 1999.

[Karaman and Frazzoli, 2011] Sertac Karaman and Emilio Frazzoli. Sampling-based algorithms for optimal motion planning. I. J. Robotics Res., 30(7):846-894, 2011.
[Kavraki et al., 1996] Lydia E. Kavraki, Petr Svestka, JeanClaude Latombe, and Mark H. Overmars. Probabilistic roadmaps for path planning in high-dimensional configuration spaces. IEEE Trans. Robotics and Automation, 12(4):566-580, 1996.

[LaValle and Kuffner, 1999] Steven M. LaValle and James J. Kuffner. Randomized kinodynamic planning. In IEEE Int. Conf. on Robotics and Automation (ICRA), pages 473479, 1999.

[LaValle, 2006] Steven M. LaValle. Planning Algorithms. Cambridge University Press, 2006.

[Lozano-Perez, 1983] Tomas Lozano-Perez. Spatial planning: A configuration space approach. IEEE Transactions on Computers, C-32(2):108-120, 1983.

[Mandalika et al., 2018] Aditiya Mandalika, Oren Salzman, and Siddhartha S. Srinivasa. Efficient shortest-path algorithm for graphs with expensive edge evaluation via lazy lookahead. In Int. Conf. on Automated Planning and Scheduling (ICAPS), pages 476-484, 2018.

[Mandalika et al., 2019] Aditiya Mandalika, Sanjiban Choushury, Oren Salzman, and Siddhartha S. Srinivasa. Generalized lazy search for robot motion planning: Interleaving search and edge evaluation via event-based toggles. In Int. Conf. on Automated Planning and Scheduling (ICAPS), 2019. to appear.

[Salzman and Halperin, 2015] Oren Salzman and Dan Halperin. Asymptotically-optimal motion planning using lower bounds on cost. In IEEE Int. Conf. on Robotics and Automation (ICRA), pages 4167-4172, 2015. 